\title{
Impurity influence in quantum spin Hall transport
}

\author{
Hsin-Han Lee, Jiun-Yue Liu, and Ching-Ray Chang \\ Department of Physics, National Taiwan University, Taipei 10617, Taiwan
}

Shun-Qing Shen

Department of Physics, The University of Hong Kong, Pokfulam Road, Hong Kong

(Received 1 July 2013; revised manuscript received 14 October 2013; published 25 November 2013)

\begin{abstract}
Influences of impurities on the electron density and transmission of a finite $\mathrm{HgTe} / \mathrm{CdTe}$ quantum well sample are studied numerically in the framework of the Landauer-Büttiker formula. In a geometry of a slab with finite width, electrons in helical edge states protected by the time-reversal symmetry can tunnel through nonmagnetic impurities at two resonant energy levels. Electrons of one side can tunnel to the other side and the quantized conductance can be broken down. For a small sample with impurity, the transmission coefficient can even drop to zero for the crosswalk between the helical edge states at two sample sides. The distance between helical edge states is critical for the suppression of the transmission. The $s$ orbital and $p$ orbital of pseudospins affect the quantized spin Hall current in a different way for their different energies.
\end{abstract}

DOI: 10.1103/PhysRevB.88.195149

PACS number(s): 73.23.-b, 73.20.At, 72.25.Dc

\section{INTRODUCTION}

Recently, topological insulators have attracted intense attention for their novel physics phenomena in materials. ${ }^{1-3} \mathrm{~A}$ two-dimensional (2D) topological insulator was first predicted in 2005 and several possible materials were proposed. This phenomenon is also called the quantum spin Hall effect and the existence of helical edge states is a signature for the quantum transport. ${ }^{4}$ The edge states of the quantum spin Hall effect are helical because their spin direction and momentum are locked with each other, and electrons of spin-up and spin-down propagate along the opposite directions on the boundary of the bulk for the time-reversal symmetry [Fig. 1(a)]. In clean systems of all kinds of materials, the magnitude of the spin Hall conductivity reaches a universal constant $e / 4 \pi$. In their initial proposal, Kane and Mele proposed that the intrinsic spin-orbit interaction within zigzag graphene nanoribbon revises the bulk band structure and induces the Dirac cone of the edge state. However, the intrinsic spin-orbit coupling in graphene is very tiny $^{5}$ and makes the quantum spin Hall effect difficult to be realized or observed on graphene. ${ }^{6}$ In 2006, the quantum spin Hall effect was predicted in a $2 \mathrm{D} \mathrm{HgTe}$ quantum well with a $\mathrm{CdTe} / \mathrm{HgTe} / \mathrm{CdTe}$ sandwich structure. ${ }^{7}$ The $\mathrm{CdTe} / \mathrm{HgTe} / \mathrm{CdTe}$ system has a strong intrinsic spin-orbit interaction reported experimentally. ${ }^{8}$ In a dirty system, for the protection of fundamental physical laws, the time-reversal symmetry makes edge states robust against nonmagnetic defect ${ }^{9,10}$ and weak interaction. $^{11,12}$ The spin-polarized electrons do not scatter backward by the defect for the time-reversal symmetry and thus two-terminal conductivity of the electron transmission is $2 e^{2} / h$ for spin-up and spin-down branches along the edges. ${ }^{10}$ Nonzero but not exactly quantized conductance in a band-insulating region has been observed ${ }^{8}$ in a $\mathrm{HgTe} / \mathrm{CdTe}$ quantum well. The intrinsic spin Hall effect ${ }^{13,14}$ of a dirty sample with nonmagnetic impurities has been intensively studied. For an infinitesimal impurity density of a dirty sample, the analytic calculations concluded a cancellation of the effect. ${ }^{15}$ Numerical analysis of mesoscopic system with finite size $^{16,17,18}$ showed that the spin Hall effect persists up to a certain impurity density (the spin Hall current is produced by the spin-orbit interaction in 2D systems or semiconductors ${ }^{19}$ ). It was also reported that the quantum spin Hall phase can be completely destroyed due to the quantum percolation of the impurity bands within the gap of bulk bands and the localization-delocalization transition is accompanied by the vanishing of the quantum spin Hall phase. ${ }^{20}$ However, the detailed mechanism of delocalization and the nonquantization of spin Hall conductance remains unclear.

There are several possible paths which electrons of topological insulators with impurity can possibly go through. Two different kinds of paths are shown in Fig. 1 and all possible paths obey the time-reversal symmetry. The first one was discussed by Qi and Zhang. ${ }^{7,8,10}$ In Fig. 1(b), electrons from edge states do not interact with impurity and the quantization of spin Hall conductance should be followed for this kind of electrons. A second possible path is that the electrons from the edge state can interact with the impurity but cannot crosswalk with another branch of the edge. Therefore, it will tunnel in and out of the impurity; however, the time-reversal symmetry requires that electrons of the helical edge state can move forward. For the second path in Fig. 1(c) electrons tunnel from edge states to bound states of impurity and back to the original edge states; there is possibly a phase shift induced from tunneling. Therefore, interference in Fig. 1(b) and Fig. 1(c) can reduce the spin Hall conductance and the reduction of transmission in Figs. 6(a), 6(b), 7(a), and 7(b) should result from the interference of two different kinds of forward electrons. The third possible path in Fig. 1(d) is a tunneling path from one side to the other side of the sample edges; the time-reversal symmetry requires the electrons to tunnel backward to the different helical edge state.

The robustness of quantum helical edge states of spin-up and spin-down electrons is expected to be useful for the new generation of nanodevices. In particular, the quantum spin Hall effect changes a topological insulator into an intrinsic spin filter without magnetic field. Therefore, the topological insulator enables several novel applications in both spintronic devices and quantum computers. Recent studies 
(a)

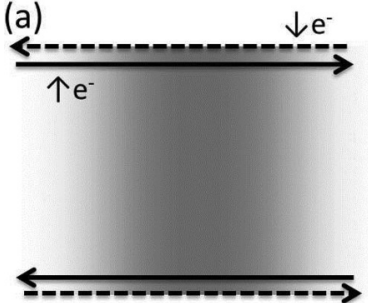

(c)

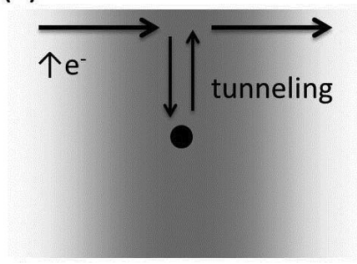

(b)

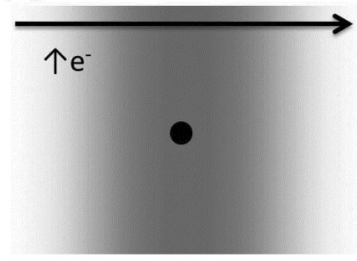

(d)

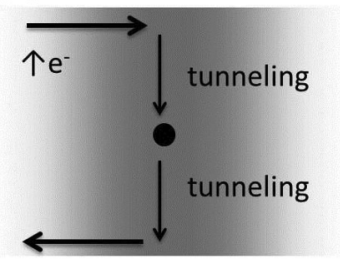

FIG. 1. (a) Helical edge-state currents of the topological insulator. (b) The edge-state current does not interact with impurity. (c) The edge-state current tunnels to the impurity bound state and tunnels back to the original edge. (d) The edge-state current tunnels to the impurity bound state and tunnels to the opposite edge state. To clear the picture, we only show the one-channel spin-up electron path with impurity; the other channel and spin follow the symmetry path.

also reveal that Majorana fermions can possibly exist at the interface between topological insulators and superconductors with ferromagnetism. ${ }^{21}$ Nevertheless, transport measurement of the edge current is an important signature in the experiment. Until now most experimental evidences reported in 2D and 3D topological insulators are mostly focused only on energy spectrum, ${ }^{22-25}$ and only a few experimental evidences are reported related to the transport of spin current. ${ }^{8}$ Some analysis indicated that the impurities within a topological insulator can induce a bound state near the helical edge states and thus affect the transport of the electron spins. ${ }^{20}$

In this paper we applied the Green's function technique ${ }^{26}$ to study electron transport in the HgTe quantum well. A HgTe quantum well is a reported topological insulator with both edge energy dispersion and transport phenomenon. ${ }^{8}$ In Sec. II, we briefly introduce the Green's function method combined with tight-binding approaches to the 2D Hamiltonian of $\mathrm{HgTe} / \mathrm{CdTe}$ quantum well. ${ }^{27}$ We introduce a localized nonmagnetic impurity to study the size effect of the 2D topological insulator in Sec. III. Two bound states of localized impurity are found from the superposition of $s$ orbitals and $p$ orbitals of electrons for either an attractive or a repulsive impurity potential. For a large size of $\mathrm{HgTe} / \mathrm{CdTe}$, the two branches of helical edge states at two sides of a sample act independently and also does not interact with localized impurity and the two-terminal conductance is always quantized to be $2 e^{2} / h$. However, for a small size of $\mathrm{HgTe} / \mathrm{CdTe}$, even a single nonmagnetic impurity affects the transport properties for the crosswalk between two different branches of helical edge states. The bound state induced by the impurity provides a tunneling channel between the helical edge states on different sides. The localization-delocalization transition at two resonance bound states accompany the loss of quantization of the conductance. It is worth pointing out that the attractive impurity potential usually induces a more significant influence on the dislocation

than repulsive impurity potential. We conclude in our analysis that defect can cause loss of quantization of quantum spin Hall conductance via the crosswalk between helical edge states. Localized bound states with two bound energies of attractive and repulsive impunity potentials are identified. When the resonance tunneling between the helical edge states occurs, the nonquantization of the conductance can be observed.

\section{NONEQUILIBRIUM TRANSPORT FORMALISM FOR TOPOLOGICAL INSULATOR}

The Hamiltonian for the HgTe quantum well can be expressed in a block-diagonal form: ${ }^{7}$

$$
\mathbf{H}=\left[\begin{array}{cc}
\mathbf{h} & 0 \\
0 & \mathbf{h}^{*}
\end{array}\right] .
$$

In this Hamiltonian, the upper $2 \times 2$ block is expanded by two pseudo-spin-up states $|s, \uparrow\rangle$ and $\left|p_{x}+i p_{y}, \uparrow\right\rangle$, and the lower block is for pseudo-spin-down states $|s, \downarrow\rangle$ and $\left|-p_{x}+i p_{y}, \downarrow\right\rangle$. In the tight-binding approximation, the Hamiltonian can be expressed as

$$
\begin{aligned}
\mathbf{H}_{T B}= & \sum_{\mathbf{i}} \varphi_{\mathbf{i}}^{\dagger}\left[\begin{array}{cccc}
E_{i s} & 0 & 0 & 0 \\
0 & E_{i p} & 0 & 0 \\
0 & 0 & E_{i s} & 0 \\
0 & 0 & 0 & E_{i p}
\end{array}\right] \varphi_{\mathbf{i}} \\
& +\sum_{\mathbf{i}} \varphi_{\mathbf{i}}^{\dagger}\left[\begin{array}{cccc}
V_{s s} & V_{s p} & 0 & 0 \\
-V_{s p}^{*} & V_{p p} & 0 & 0 \\
0 & 0 & V_{s s} & V_{s p}^{*} \\
0 & 0 & -V_{s p} & V_{p p}
\end{array}\right] \varphi_{\mathbf{i}+\delta \mathbf{x}}+\text { H.c. } \\
+ & \sum_{\mathbf{i}} \varphi_{\mathbf{i}}^{\dagger}\left[\begin{array}{cccc}
V_{s s} & i V_{s p} & 0 & 0 \\
i V_{s p}^{*} & V_{p p} & 0 & 0 \\
0 & 0 & V_{s s} & -i V_{s p}^{*} \\
0 & 0 & -i V_{s p} & V_{p p}
\end{array}\right] \varphi_{\mathbf{i}+\delta \mathbf{y}}+\text { H.c. }
\end{aligned}
$$

by introducing the spinor $\varphi=\left[c_{s, \mathbf{i}}^{\uparrow}, c_{p, \mathbf{i}}^{\uparrow}, c_{s, \mathbf{i}}^{\downarrow}, c_{p, \mathbf{i}}^{\downarrow}\right]^{T}$. Here the index $\mathbf{i}$ represents the lattice site in the real space and $\delta x$ and $\delta y$ are unit vectors along the $x$ and $y$ directions, respectively. Those parameters for on-site energy are $E_{s}=C+M-$ $4(B+D) / a^{2}$ and $E_{p}=C-M-4(D-B) / a^{2}$. For the hopping energy term $V_{s p}=-i A / 2 a, V_{s s}=(B+D)=a^{2}$, $V_{p p}=(D-B) / a^{2}$. Here $a$ is the lattice constant chosen as $5 \mathrm{~nm}$ and the other parameters are $A=364.5 \mathrm{meV} \mathrm{nm}$, $B=-686 \mathrm{meV} \mathrm{nm}^{2}$, and $C=0, D=-52 \mathrm{meV} \mathrm{nm}^{2}$. The value of $M$ is a function of thickness of the quantum wells which decides whether the material is topologically trivial or nontrivial. ${ }^{27}$ Here we choose $M=-10 \mathrm{meV}$ for a topological insulator. These parameters are controllable in experiment. ${ }^{8}$

The impurity potential in a sample is introduced by including

$$
\mathbf{W}=\sum_{\mathbf{i}} \varphi_{\mathbf{i}}^{\dagger}\left[\begin{array}{cccc}
W_{\mathbf{i}} & 0 & 0 & 0 \\
0 & W_{\mathbf{i}} & 0 & 0 \\
0 & 0 & W_{\mathbf{i}} & 0 \\
0 & 0 & 0 & W_{\mathbf{i}}
\end{array}\right] \varphi_{\mathbf{i}}
$$


where $W_{\mathbf{i}}=W$ is on the lattice site $\mathbf{i}$ in the potential area; otherwise, $W_{\mathbf{i}}=0$.

In this paper, we only consider the upper block matrix for the two pseudo-spin-up states. The contributions from the states for the pseudo-spin-down states are their counterpartners of the upper block matrix under the time reversal.

\section{Landauer formalism for nonequilibrium transport}

In this study, the geometry is infinite and it is not a periodic system because the single impurity exists. Instead of the Bloch theorem, the Landauer formalism is suitable for this calculation. First, we consider a sample connect two semi-infinite leads. The effective Hamiltonian can be written as follows. ${ }^{26}$ Here $\Sigma_{R(L)}$ is the self-energy matrix for the right (left) lead, which includes the lead effect on the sample we want to take into account, the real part makes the energy spectra shift, and the imaginary part make it broadening. The formula of the self-energy matrix is $\Sigma_{R(L)}(E)=\tau_{R(L)} g_{s, R(L)}(E) \tau_{R(L)}^{\dagger}$, where $\tau$ is the hopping matrix between the lead and the device and $g_{s}$ is the surface Green's function matrix that is calculated by the recursive relation

$$
g_{s}(E)=\left[(E+i \eta) \mathbf{I}-H_{\text {layer }}-\tau g_{s}(E) \tau^{\dagger}\right]^{-1},
$$

where the $H_{\text {layer }}$ is a one-layer Hamiltonian for the lead; the imaginary part of the value $\eta$ is a very small positive number for numerical convergence. This formula is convenient to operate but it is hard to make convergent for all systems for a computation, so the most highly convergent scheme for the calculation should be used. ${ }^{28}$ The retarded Green's function matrix for the device connecting to two leads is given:

$$
\mathbf{G}^{r}(E)=\left[(E+i \eta) \mathbf{I}-\mathbf{H}_{\text {effect }}(E)\right]^{-1} .
$$

The local density of states is obtained from the retarded Green's function matrix

$$
\left\langle N_{m ; \sigma, n}(E)\right\rangle=-\frac{1}{\pi} \operatorname{Im}\left[\mathbf{G}_{m, m ; \sigma, n}^{r}(E)\right]
$$

for electrons with spin $\sigma$ and the orbital $n$ for the energy $E$ in the site $m$. The transmission coefficient (or conductance) can be calculated from the Landauer-Büttiker formalism:

$$
T_{L R}(E)=\operatorname{Tr}\left[\Gamma_{L}(E) G^{r}(E) \Gamma_{R} G^{r \dagger}(E)\right] .
$$

The conductance is obtained by multiplying the transmission $T_{L R}(E)$ by the conductance unit $e^{2} / h$. Here $\Gamma_{L(R)}$ is the broadening matrix for the right (left) lead. This matrix is related the imaginary part of the self-energy matrix; the formula is

$$
\Gamma_{L(R)}=i\left[\Sigma_{R(L)}-\Sigma_{R(L)}^{\dagger}\right]
$$

\section{TOPOLOGICAL INSULATOR WITH IMPURITY}

In this study, we consider an infinitely long sample (along the $x$ direction) of a finite width $L_{y}$ with a nonmagnetic impurity of $5 \times 5 \mathrm{~nm}^{2}$ located at $x=0$. The position of the impurity along the $y$ axis can be different: at the center of the sample or closer to one of the side boundary. In the practical calculation, we divide the sample into three parts: The central part contains the impurity potential with a width $L_{x}$ larger than the potential regime, and the left and right parts are regarded (a)

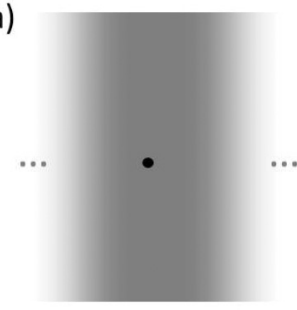

(c)

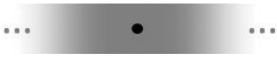

(b)

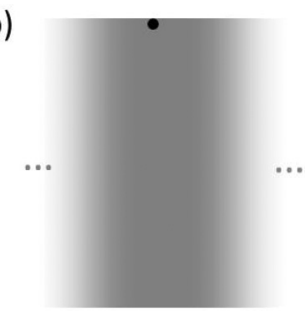

(d)

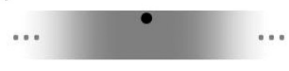

FIG. 2. An impurity located in the HgTe quantum well sample. (a),(b) $L_{y}=2005 \mathrm{~nm}$; (c),(d) $L_{y}=295 \mathrm{~nm}$.

as two semi-infinite leads as introduced in Sec. II B. After numerical calculations we found that the numerical results in the present study are insensitive to the values of $L_{x}$, as anticipated, and thus we take $L_{x}=15 \mathrm{~nm}$ in the calculations. The Fermi energy is set to be $E_{F}=8.74 \mathrm{meV}$ at the Dirac cone of helical edge states if there is no specific indication.

We first plot the transmission with a series of different width $L_{y}$ by means of the Landauer-Büttiker formalism[Eq. (7)] and decide the width for the further calculation in the absence of the impurity, i.e., $W=0$. Then we add a single nonmagnetic impurity with dimension of $5 \times 5 \mathrm{~nm}^{2}$ to the sample region. We decide the impurity affect by varying the impurity potential $W$ in Eq. (3). In the calculation we take several specific values within the range of $(-0.5 \leqslant W \leqslant 0.5)$ and show the nontrivial range in Figs. 4-7. Two samples with different dimensions are analyzed to study the size effect on the defect-induced localization-delocalization transition. For simplicity we study two cases of $L_{y}=2005$ and $295 \mathrm{~nm}$. We also take into additional account the locations of the impurity. Two different situations are considered within all samples: a central defect between two edges and an edge defect near the upper edge (see Fig. 2). Two clean samples are also calculated for comparison. Thus, direct tunneling between the helical edge states at two sides from the finite-size effect and the indirect tunneling through the impurity can be easily distinguished.

\section{A. Clear limit of two samples}

We first study two samples without the impurity. Here we studied several different widths to understand the size effect in the clean case and we did not observe loss of quantization spin Hall conductance until a width of less than $120 \mathrm{~nm}$, as shown in Fig. 3(a). There was a sharp transition from one to zero in the clean limit for narrow-width sample, which is expected as the finite-size effect. ${ }^{29}$ In this calculation the Fermi level is fixed at $E_{F}=8.47 \mathrm{meV}$. If we plot the transmission coefficient as a function of the Fermi energy as shown in Fig. 3(b), for $L_{y}=295 \mathrm{~nm}$, the transmission coefficient drops to zero at a finite range near $E_{F}=7.5 \mathrm{meV}$; for $L_{y}=120 \mathrm{~nm}$, the transmission is equal to zero at $E_{F}=7.5 \mathrm{meV}$ in a rather wide energy interval. This demonstrates an energy gap opening near the crossing point of energy dispersion of helical edge states. 
(a)

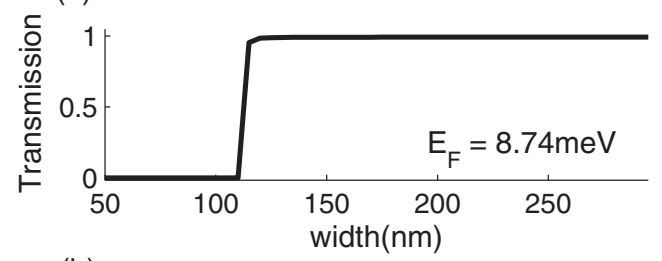

(b)

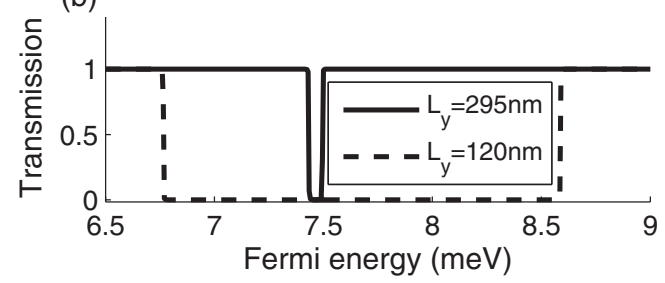

FIG. 3. (a) The transmission coefficient as a function of the sample width for the Fermi energy is $E_{F}=8.74 \mathrm{meV}$. (b) The transmission coefficient as a function of the Fermi energy $E_{F}$ for $L y=295$ and $120 \mathrm{~nm}$.

\section{B. In-gap bound states induced by the impurity}

In the calculation, we found the formation of two in-gap bound states induced by the impurity. The two bound states have positive and negative energies, respectively. This result is computed by the local density of state (6), and the unit is $(1 / \mathrm{eV})$.

For an attractive impurity potential of $W=-439.2 \mathrm{meV}$, electrons are likely to jump into the defect region. The bound-state energy is $E_{-}=-439.2 \mathrm{meV}$ for the impurity in the center [Figs. 2(a) and 2(c)] and $E_{-}=-328.5 \mathrm{meV}$ for the impurity on the edge [Figs. 2(b) and 2(d)]. $|s, \uparrow\rangle$ electrons will form an isotropic and circular in-gap bound

(a)
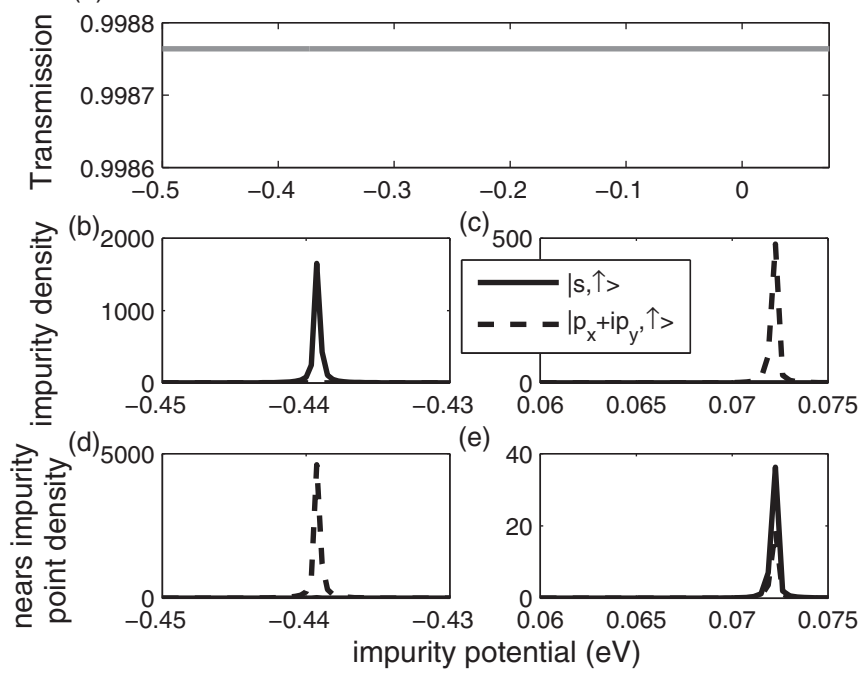

FIG. 4. $L=2005 \mathrm{~nm}$, the impurity in the center of the slab $(y=0)$. (a) The transmission coefficient of topological insulator with the impurity is the same with different amplitude $W$ of the impurity potential. (b) The charge density on the impurity site with an attractive potential $(W<0)$. (c) The charge density on the impurity site with a positive potential $(W>0)$. (d) The charge density around the impurity site with attractive potential $(W<0)$. (e) The charge density around the impurity site with a positive potential $(W>0)$.

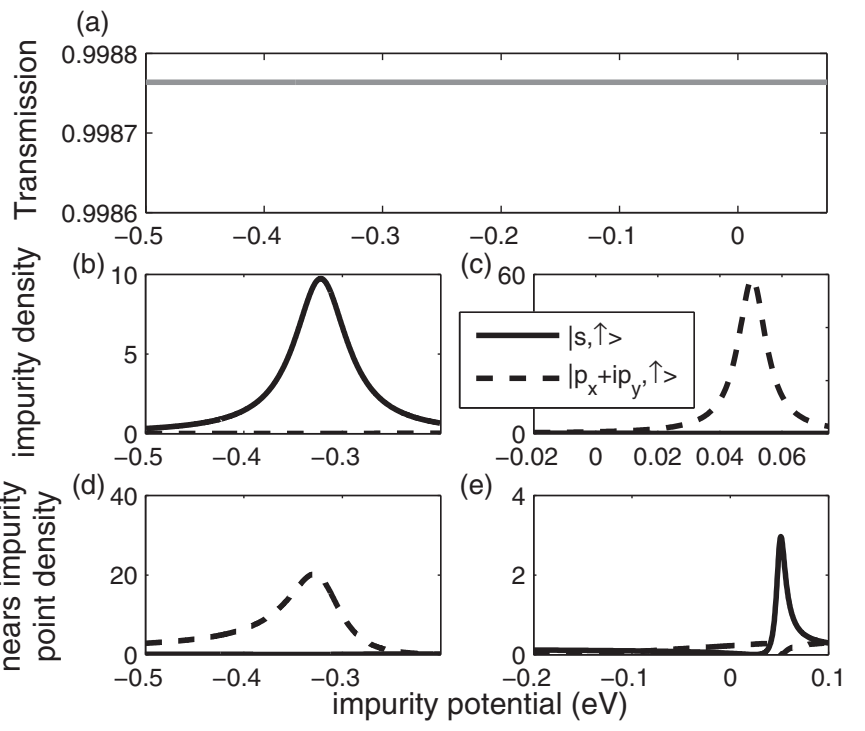

FIG. 5. $L_{y}=2005 \mathrm{~nm}$, the impurity located on the edge ( $y=$ $1000 \mathrm{~nm}$ ). (a) The transmission coefficient of topological insulator with the impurity is the same with different amplitude $W$ of the impurity potential. (b) The charge density on the impurity site with an attractive potential $(W<0)$. (c) The charge density on the impurity site with a positive potential $(W>0)$. (d) The charge density around the impurity site with attractive potential $(W<0)$. (e) The charge density around the impurity site with a positive potential $(W>0)$.

state near the defect site, while $\left|p_{x}+i p_{y}, \uparrow\right\rangle$ electrons are form a ring structure outside the $|s, \uparrow\rangle$ electron circle. The low energy $|s, \uparrow\rangle$ is within the quantum well, while the $\left|p_{x}+i p_{y}, \uparrow\right\rangle$ electrons are circulating around the potential well [Figs. 4(b), 4(d), 5(b), 5(d), 6(c), 6(e), 7(c), and 7(e)].

For a repulsive impurity potential of $W=+72.2 \mathrm{meV}$, a new boundary is formed to attract surrounding electrons as the formation of the edge states near the boundary. The bound-state energy $E_{+}=0.0722,0.0516,0.0719$, and $0.0516 \mathrm{eV}$ for Figs. 2(a)-2(d), respectively. $\left|p_{x}+i p_{y}, \uparrow\right\rangle$ electrons form a in-gap bound state with circular distribution within the defect, while the low-energy $|s, \uparrow\rangle$ electrons accumulates as a ringlike structure outside the defect [Figs. 4(c), 4(e), 5(c), 5(e), 6(d), 6(f), 7(d), and 7(f)]. Although the spatial distributions of $\left|p_{x}+i p_{y}, \uparrow\right\rangle$ and $|s, \uparrow\rangle$ are very different in the in-gap bound states with positive and negative energy, $\left|p_{x}+i p_{y}, \uparrow\right\rangle$ and $|s, \uparrow\rangle$, electrons resonate at the same energy. A similar phenomenon was reported for various dimensions of the topological insulator including a $\mathrm{HgTe}$ quantum well in a continuous model. ${ }^{30}$

From our calculations, we also noted that the position of impurity plays an important role. When an impurity is on the edge, as shown in Figs. 2(b) and 2(d), the localized bound state will interfere with the helical edge state and thus the width of the localized bound state becomes broadening [Figs. 5(b)5(e), 7(c)-7(f)]. However, when an impurity is in the center of the bulk in Figs. 2(a) and 2(c), the interference between the helical edge state and the bound state are much less than the edge defect and thus the width broadening effect of localized bound states drops significantly. In summary, broadening of the localized bound state critically depends on the distance between impurity and the boundary of the sample. 


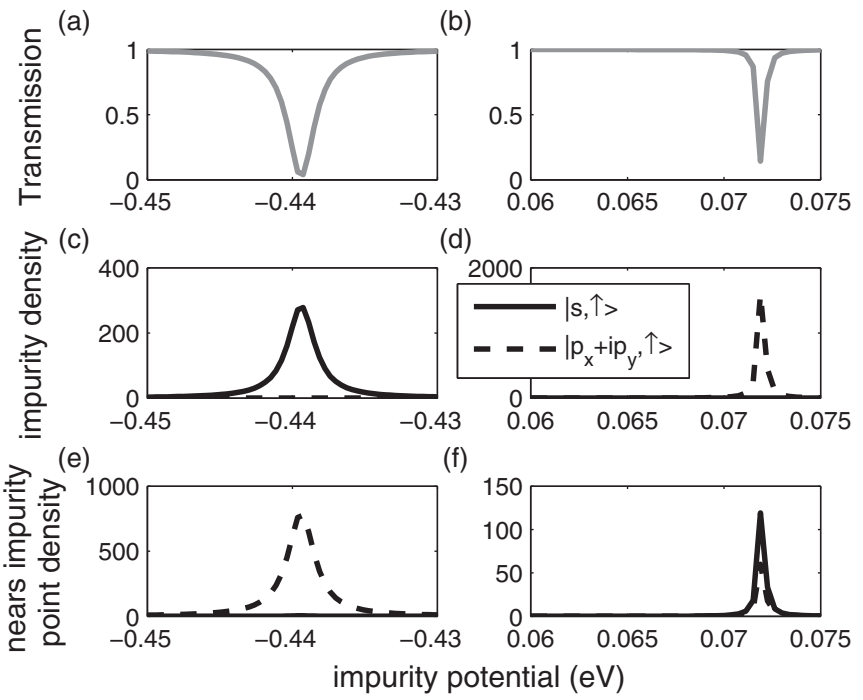

FIG. 6. $L_{y}=295 \mathrm{~nm}$, the impurity located in the center $(y=0)$. (a) The transmission coefficient of topological insulator with the impurity is the same with different amplitude $W$ of the impurity potential. (b) The charge density on the impurity site with an attractive potential $(W<0)$. (c) The charge density on the impurity site with a positive potential $(W>0)$. (d) The charge density around the impurity site with attractive potential $(W<0)$. (e) The charge density around the impurity site with a positive potential $(W>0)$.

\section{TRANSMISSION OF ELECTRONS}

For the sample with its width $L_{y}=2005 \mathrm{~nm}$, the relation between the transmission coefficient and the impurity potential $W$ is plotted in Figs. 4(a) and 5(a) for different positions of the impurity. The charge densities within the impurity area
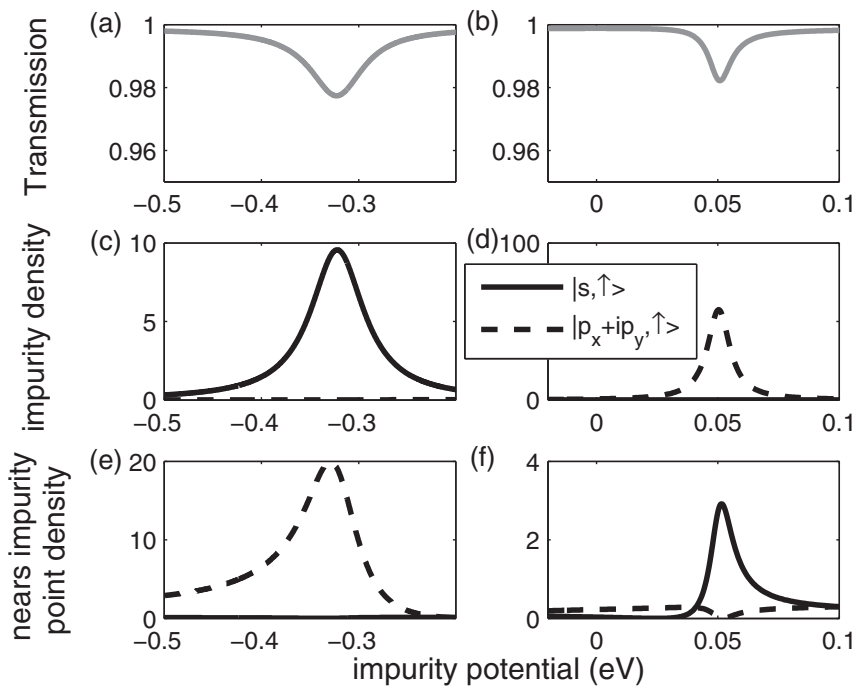

FIG. 7. $L_{y}=295 \mathrm{~nm}$, the impurity located on edge ( $y=$ $145 \mathrm{~nm}$ ). (a) The transmission coefficient of topological insulator with the impurity is the same with different amplitude $W$ of the impurity potential. (b) The charge density on the impurity site with an attractive potential $(W<0)$. (c) The charge density on the impurity site with a positive potential $(W>0)$. (d) The charge density around the impurity site with attractive potential $(W<0)$. (e) The charge density around the impurity site with a positive potential $(W>0)$. near the edge are quite different from those at the center. The energy for the bound states will shift with the location of impurities. Even though there are in-gap bound states of impurity, the transmission coefficients of electrons along the helical edge states are not influenced by the bound states at all. The behavior of transmission is exactly identical with that in a clean HeTe quantum well.

For the sample of $L_{y}=295 \mathrm{~nm}$, we observe the finite-size effect with impurity, which is actually consistent with the results of the clean case. The interacting range of helical edge states are around $120 \mathrm{~nm}$ [Fig. 3]. The transmission reduces at two specific impurity potentials [Figs. 6(a), 6(b), 7(a), and $7(b)]$; the effect is more apparent especially when the impurity locates at the center [Figs. 6(a) and 6(b)].

The transmission reduction also depends on the sample width. Both the edge states and the bound states of impurity have finite spatial distributions. If the wave functions of two states have overlap in space, electrons in these states have the probability to move from one state to another. In our calculation with adapted model parameters, if the width $L_{y}<1005 \mathrm{~nm}$, the impurity at the center may provide transverse channels connecting between helical edge states at two different sites, and thus the electrons of edge states can tunnel from one side to the other side of the sample and the backscattering occurs. This effect is quite similar to the quantum percolation in quantum spin Hall antidot systems. ${ }^{20}$ It is worth noting that the backward scattering here does not violate the general argument from the time-reversal symmetry: The symmetry does not prohibit in the backward scattering within two helical edge states through the impurity. ${ }^{10}$ Therefore, the transmission of a small topological insulator sample with impurity can even drop to zero at the specific energy of the impurity band [Figs. 6(a) and 6(b)]. The local density of states for the $L=295 \mathrm{~nm} \mathrm{HgTe}$ quantum well is plotted in Fig. 8; it is consistent with the result of Fig. 6. (a)

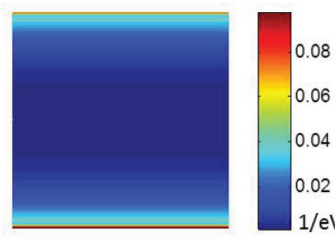

(c)

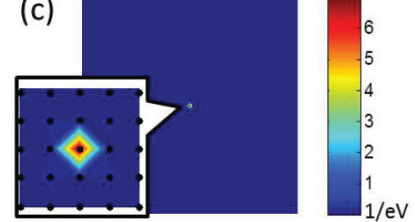

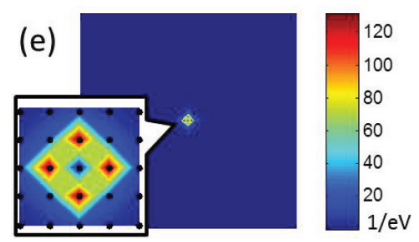

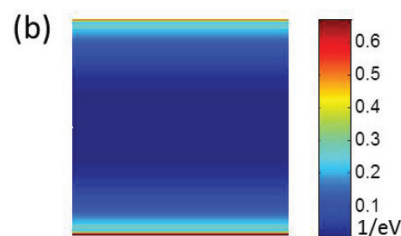

(d)
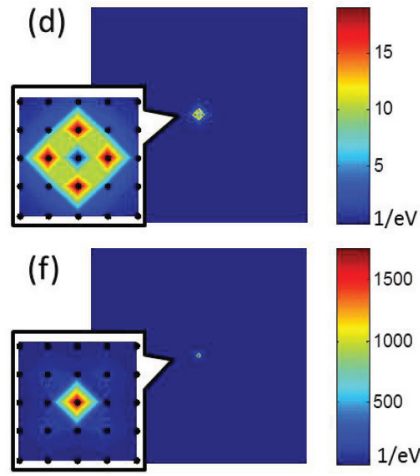

FIG. 8. (Color online) The local density of state for the $L_{y}=$ $295 \mathrm{~nm} \mathrm{HgTe}$ QW, $E_{F}=8.74 \mathrm{meV}$. (a) $|s, \uparrow\rangle$ state in a clear system. (b) $\left|p_{x}+i p_{y}, \uparrow\right\rangle$ state in a clear system. (c) $|s, \uparrow\rangle$ state at $W=$ $-439.2 \mathrm{meV}$. (d) $\left|p_{x}+i p_{y}, \uparrow\right\rangle$ state at $W=71.9 \mathrm{meV}$. (e) $|s, \uparrow\rangle$ state at $W=-439.2 \mathrm{meV}$. (f) $\mid p_{x}+i p_{y}$, $\left.\uparrow\right\rangle$ state at $W=71.9 \mathrm{meV}$. 


\section{DISCUSSIONS AND CONCLUSIONS}

Our studies indicate that even though the helical edge states are robust against small nonmagnetic impurity from the protection of time-reversal symmetry, the quantized spin Hall conductance is quite sensitive to the impurity in a finite-size sample. Transmission of the robust helical states can be reduced from the crosswalk between helical edge states at two sides. The finite-size effect of topological insulator is not only observed in the clean case, but also is significant with impurity inside the sample. Loss of quantized conductance of edge electrons depends on either the amplitude of impurity potential or the width of the sample. From our analysis, we know that nonmagnetic impurity will not destroy the helical edge states but it will induce tunneling between two helical edge states. The finite-size effect can open an energy gap on the helical edge state and direct overlapping of wave function from two helical edge states, which may let the nondissipative conducting quantum spin Hall channel disappear. Another possible reduction mechanism of quantized spin Hall conductance is from the impurity within topological insulator; impurity-induced bound states provide a crosswalk channel of helical edge states. In summary, for a quantum spin Hall nanodevice, the impurity in a $2 \mathrm{D} \mathrm{CdTe} / \mathrm{HgTe} / \mathrm{CdTe}$ system of topological insulators can change the electronic structure and also the transport features within sample. The electrons within the helical edge states can trasverse through impurity and crosswalk to other branches and this transition induces the reduction of quantization spin Hall conductance.

\section{ACKNOWLEDGMENTS}

The authors thank Kuo-Wei Chen, Chien-Liang Chen, and $\mathrm{Yu}-\mathrm{Hsin} \mathrm{Su}$ for discussions. This work is supported by Republic of China National Science Council Grant No. NSC 98-2112-M-002-012-MY3 and Research Grant Council of Hong Kong Grant No. HKU7051/11P.
${ }^{1}$ M. Z. Hasan and C. L. Kane, Rev. Mod. Phys. 82, 3045 (2010).

${ }^{2}$ X. L. Qi and S. C. Zhang, Rev. Mod. Phys. 83, 1057 (2011).

${ }^{3}$ S. Q. Shen, Topological Insulators (Springer-Verlag, Berlin, 2012).

${ }^{4}$ C. L. Kane and E. J. Mele, Phys. Rev. Lett. 95, 226801 (2005).

${ }^{5}$ H. Min, J. E. Hill, N. A. Sinitsyn, B. R. Sahu, L. Kleinman, and A. H. MacDonald, Phys. Rev. B 74, 165310 (2006).

${ }^{6}$ Y. Yao, F. Ye, X. L. Qi, S. C. Zhang, and Z. Fang, Phys. Rev. B 75, 041401(R) (2007).

${ }^{7}$ B. A. Bernevig, T. L. Hughes, and S. C. Zhang, Science 314, 5806 (2006).

${ }^{8}$ M. König, S. Wiedmann, C. Brune, A. Roth, H. Buhmann, L. W. Molenkamp, X. L. Qi, and S. C. Zhang, Science 318, 766 (2007).

${ }^{9}$ C. L. Kane and E. J. Mele, Phys. Rev. Lett. 95, 146802 (2005).

${ }^{10}$ X. L. Qi and S. C. Zhang, Phys. Today 63(1), 33 (2010).

${ }^{11}$ C. Wu, B. A. Bernevig, and S. C. Zhang, Phys. Rev. Lett. 96, 106401 (2006).

${ }^{12}$ C. Xu and J. E. Moore, Phys. Rev. B 73, 045322 (2006).

${ }^{13}$ J. Schliemann and D. Loss, Phys. Rev. B 69, 165315 (2004).

${ }^{14}$ J. Sinova, S. Murakami, S. Q. Shen, and M. S. Choi, Solid State Commun. 138, 4 (2006).

${ }^{15}$ O. V. Dimitrova, Phys. Rev. B 71, 245327 (2005).

${ }^{16}$ N. Sugimoto, S. Onoda, S. Murakami, and N. Nagaosa, Phys. Rev. B 73, 113305 (2006).

${ }^{17}$ C. P. Moca and D. C. Marinescu, Phys. Rev. B 72, 165335 (2005).

${ }^{18}$ B. K. Nikolić, S. Souma, L. P. Zârbo, and J. Sinova, Phys. Rev. Lett. 95, 046601 (2005).
${ }^{19}$ B. A. Bernevig, J. P. Hu, E. Mukamel, and S. C. Zhang, Phys. Rev. B 70, 113301 (2004); B. A. Bernevig and S. C. Zhang, ibid. 72, 115204 (2005); E. I. Rashba, ibid. 68, 241315 (2003); A. A. Burkov, A. S. Nunez, and A. H. MacDonald, ibid. 70, 155308 (2004).

${ }^{20}$ R. L. Chu, J. Lu, and S. Q. Shen, EPL 100, 17013 (2012).

${ }^{21}$ L. Fu and C. L. Kane, Phys. Rev. Lett. 100, 096407 (2008).

${ }^{22}$ D. Hsieh, D. Qian, L. Wray, Y. Xia, Y. S. Hor, R. J. Cava, and M. Z. Hasan, Nature (London) 452, 970 (2008).

${ }^{23}$ D. Hsieh, Y. Xia, D. Qian, L. Wray, J. H. Dil, F. Meier, J. Osterwalder, L. Patthey, J. G. Checkelsky, N. P. Ong, A. V. Fedorov, H. Lin, A. Bansil, D. Grauer, Y. S. Hor, R. J. Cava, and M. Z. Hasan, Nature (London) 460, 1101 (2009).

${ }^{24}$ X. Dai, T. L. Hughes, X. L. Qi, Z. Fang, and S. C. Zhang, Phys. Rev. B 77, 125319 (2008).

${ }^{25}$ Y. L. Chen, J. G. Analytis, J. H. Chu, Z. K. Liu, S. K. Mo, X. L. Qi, H. J. Zhang, D. H. Lu, X. Dai, Z. Fang, S. C. Zhang, I. R. Fisher, Z. Hussain, and Z. X. Shen, Science 325, 178 (2009).

${ }^{26}$ Supriyo Datta, Quantum Transport: Atom to Transistor (Cambridge University Press, Cambridge, UK, 2005).

${ }^{27}$ H. Jiang, L. Wang, Q. F. Sun, and X. C. Xie, Phys. Rev. B 80, 165316 (2009)

${ }^{28}$ M. P. Lopez Sancho, J. M. Lopez Sancho, J. M. L. Sancho, and J. Rubio, J. Phys. F: Met. Phys. 15, 851 (1985).

${ }^{29}$ B. Zhou, H. Z. Lu, R. L. Chu, S. Q. Shen, and Q. Niu, Phys. Rev. Lett. 101, 246807 (2008).

${ }^{30}$ J. Lu, W.-Y. Shan, H.-Z. Lu, and S.-Q. Shen, New J. Phys. 13, 103016 (2011). 